\title{
Bamboo leaf extract improves spatial learning ability in a rat model with senile dementia*
}

\author{
Jian-xiang LIU, Min-ying ZHU, Ci-yuan FENG, Hai-bin DING, Ying ZHAN, Zhan ZHAO, Yue-min DING ${ }^{\dagger *}$ \\ (Department of Clinical Medicine, School of Medicine, Zhejiang University City College, Hangzhou 310015, China) \\ ${ }^{\dagger}$ E-mail: dingyuemin@zucc.edu.cn \\ Received Sept. 20, 2014; Revision accepted Feb. 1, 2015; Crosschecked June 16, 2015
}

\begin{abstract}
Senile dementia (SD) is a syndrome characterized by progressive neurological deterioration. Treatment for the disease is still under investigation. Bamboo leaf extract (B-extract) has been known for its biological efficacy in anti-oxidant and anti-cancer activities. However, study on B-extract for its protection against dementia is very limited. The effect of B-extract on a rat model with SD was examined. B-extract improved spatial learning ability of the dementia rats. The hippocampus of dementia model rats showed reduced levels of acetylcholine (ACh), epinephrine (E), norepinephrine (NE), and dopamine (DA), and increased activities of acetylcholine esterase (AChE) and monoamine oxidase (MAO). Treatment with B-extract $20 \mathrm{mg} /(\mathrm{kg} \cdot \mathrm{d})$ for 7 weeks significantly inhibited the enzyme activity compared with untreated dementia rats, and raised the levels of $A C h, E$, and DA in the hippocampus. In addition, treatment with B-extract elevated the level of $\mathrm{Y}$-aminobutyric acid (GABA), but reduced the level of glutamate (Glu) in the brain. These data suggest that B-extract might be a potential drug in treating impairment of spatial memory in dementia rats by regulating the central neurotransmitter function.
\end{abstract}

Key words: Bamboo leaf extract, Dementia, Alzheimer's disease, Neurotransmitter doi:10.1631/jzus.B1400249 Document code: A CLC number: R285.5

\section{Introduction}

Senile dementia (SD) causes long term deficit in memory and cognition that is severe enough to affect a person's daily functioning (Sharma and Singh, 2010). It is reported that in China the number of people with dementia is 6-7 million with an incidence of $5 \%-7 \%$ of people over 65 years of age (Song and Wang, 2010). Alzheimer's disease (AD) is the most common type of dementia. It is estimated that there will be more than 80 million $\mathrm{AD}$ patients in the world by 2040 (Ferri et al., 2005) and it has become the

\footnotetext{
Corresponding author

* Project supported by the Zhejiang Provincial Natural Science Foundation of China (No. LY14H090002) and the National Undergraduate Training Programs for Innovation and Entrepreneurship of China (No. 201313021016)

(D) ORCID: Yue-min DING, http://orcid.org/0000-0002-0763-9400

(C) Zhejiang University and Springer-Verlag Berlin Heidelberg 2015
}

fourth largest fatal disease (Hoyert and $\mathrm{Xu}, 2012$ ). Treatment costs of AD patients amount to 604 billion US dollars per year in the world (Wimo et al., 2013). Such huge costs have caused a great impact on social and economic development.

However, at present, the etiology and disease progression of $\mathrm{AD}$ is not fully understood. The main hypotheses are as follows: apoptosis of the central cholinergic neurons and reduction of cholinergic neurotransmitters (Xie et al., 2006; Wang and Ding, 2008; Checler and Turner, 2012), aggregation and deposition of amyloid- $\beta$ (A $\beta$ ) (Hardy, 2006), abnormal phosphorylation of tau protein (Lewczuk et al., 2004; Iqbal et al., 2005; Chun and Johnson, 2007), and formation of a neurofibrillary tangle. In addition, theories such as neurotoxicity of the excitatory neurotransmitter, oxidative stress, and mitochondrial damage can also explain the causes of dementia to some extent and provide a new theoretical basis and 
therapeutic target for the development of novel drugs for AD (Tamagno et al., 2006; Liu et al., 2010). Current medications commercially available for the treatment of $\mathrm{AD}$ can improve cognitive ability and daily behavior of AD patients (Deguil et al., 2013). However, these drugs can only alleviate the symptoms of patients instead of terminating or fundamentally reversing the dementia process (Wermuth, 2004).

Bamboo leaf is used to treat many diseases in Chinese traditional medicine. In recent years, studies have found that bamboo leaf extract (B-extract) has excellent biological efficacy in anti-oxidant activity, anti-aging activity, resistance to free-radical, and cardiovascular protection (Lee et al., 2008; Choi et al., 2013). Theoretically, these functions would have a therapeutic effect for AD. However, study on B-extract for its protection against neurodegenerative diseases and resistance to dementia is very limited. In this project, a rat model with SD was obtained by intracerebroventricular injection of amyloid peptide after chronic intraperitoneal injections of D-galactose (D-gal) for 6 weeks (Wei et al., 2005). During the period, the rats were fed with B-extract via gastric gavage for 7 consecutive weeks. Evaluation of behavioral and biochemical parameters was conducted to systematically study the effect of B-extract on the spatial cognitive abilities of dementia model rats and the underlying mechanisms.

\section{Materials and methods}

\subsection{Ethics statement}

The experimental procedures were approved by the Animal Ethics Committee of Zhejiang University (Hangzhou, China) and were carried out in accordance with institutional guidelines. All efforts were made to minimize the number of animals used and their suffering.

\subsection{Reagents}

B-extract was purchased from Johncan Biotechnology (Hangzhou, China). D-gal was purchased from Changqing Chemical (Hangzhou, China). $\mathrm{A} \beta_{1-42}$ was purchased from GL Biochem (Shanghai, China). Enzyme-linked immunosorbent assay (ELISA) kits for acetylcholine (ACh), acetylcholine esterase (AChE), choline acetyltransferase (ChAT), monoamine oxidase (MAO), dopamine (DA), epinephrine (E), norepinephrine (NE), 5-hydroxy tryptamine (5-HT), glutamate (Glu), and $\gamma$-aminobutyric acid (GABA) were purchased from Jingtian Bio-Tech (Shanghai, China).

\subsection{Animal groups}

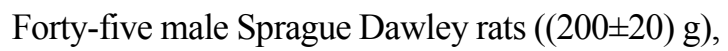
purchased from SLAC Laboratory (Shanghai, China), were randomly divided into three groups: control group, SD model group (SD), and bamboo leaf extract treatment group (B-extract $+\mathrm{SD}$ ), with 15 rats in each group. The rats in the $\mathrm{SD}$ and $\mathrm{B}$-extract $+\mathrm{SD}$ groups were injected intraperitoneally with D-gal $(60 \mathrm{mg} /(\mathrm{kg} \cdot \mathrm{d}))$ during the first 6 weeks to induce aging models and the rats in the control group were injected with the same volume of saline. At the same time, the rats in the treatment group were fed with B-extract $(20 \mathrm{mg} /(\mathrm{kg} \cdot \mathrm{d}))$ via gastric gavage, while the rats in the control group and the SD group were fed with equal amounts of physiological saline. At the end of the 6th week, the rats in the SD and B-extract+SD groups were anaesthetized with intraperitoneal injection of $2 \%(0.02 \mathrm{~g} / \mathrm{ml})$ of pentobarbital sodium $(80 \mathrm{mg} / \mathrm{kg})$. A stereotaxic apparatus (Stoelting, USA) was used to accurately position right lateral ventricle $(1.0 \mathrm{~mm}$ behind anterior fontanelle, $1.5 \mathrm{~mm}$ right to raphe, and $3.5 \mathrm{~mm}$ under cortical surface), into which $5 \mu$ of $\mathrm{A} \beta_{1-42}(2 \mu \mathrm{g} / \mu \mathrm{l})$ was slowly injected (Lamberty et al., 1992). After that, the rats were fed with B-extract or saline by gastric gavage continuously for one more week before behavioral testing (Fig. 1).
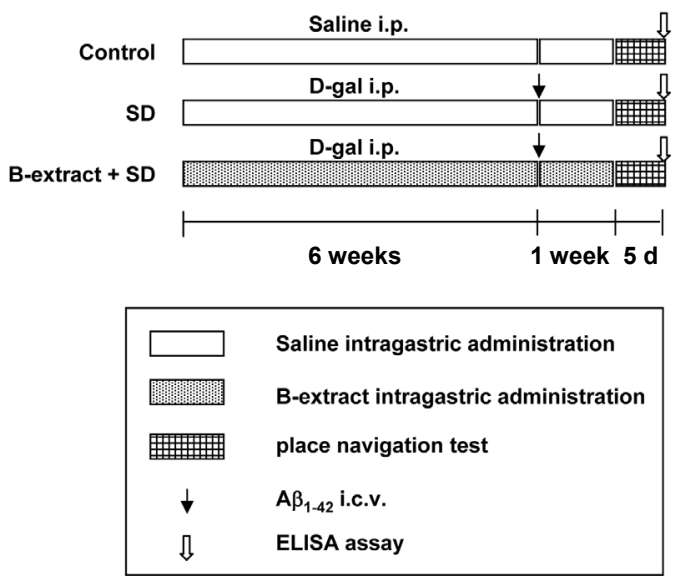

Fig. 1 Timeline of experimental protocol i.p.: intraperitoneal injection; i.c.v.: intracerebroventricular injection 


\subsection{Morris water maze test}

After seven weeks of B-extract treatment, all animals were tested for place navigation with the Morris water maze (RWD Life Science, Shenzhen, China) for five consecutive days. The rats were put into the water four times a day from different quadrants. The time that a rat took to find the platform (escape latency) was recorded. The experimental process was recorded by video and analysis software of small animal behavior (SMART 2.5, Harvard, USA) was used to determine escape latency.

\subsection{Neurotransmitter detection}

Immediately after behavioral testing, the animals were anaesthetized and decapitated. Hippocampus and cortical tissues of the rats were quickly dissected out and homogenized with ultrasound and diluted with saline. The ELISA method was applied to determine the biochemical parameters and neurotransmitter content. The operation was performed according to the instructions of the kit. Briefly, the supernatant was taken out after centrifugalization at $12000 \mathrm{r} / \mathrm{min}\left(4^{\circ} \mathrm{C}\right)$ for $30 \mathrm{~min}$, and the contents of $\mathrm{ACh}$, DA, E, NE, 5-HT, Glu, and GABA as well as the activities of AChE, ChAT, and MAO were determined.

\subsection{Statistical analysis}

Data were expressed as mean \pm standard error of the mean (SEM) and analyzed using SPSS 13.0 statistical software (SPSS, Chicago, IL, USA). Comparisons between groups were analyzed by two-way analysis of variance (ANOVA) or one-way ANOVA and Dunnett's test. A level of $P<0.05$ was considered statistically significant.

\section{Results}

\subsection{Effects of B-extract on spatial learning and memory of dementia rats}

A rat model with SD was established and the dementia rats were fed with B-extract for 7 weeks. At the end of the 7 th week, the rats were tested for spatial learning and memory with a 5-d Morris water navigation task. Subsequently, the average escape latency and path length between the point of origin and platform were measured every day (Fig. 2). As a result, the difference of the average latency and path length among the three groups on the first day of the test was not statistically significant $(P>0.05)$. After training for two days, average escape latency and path length (a)

(b)
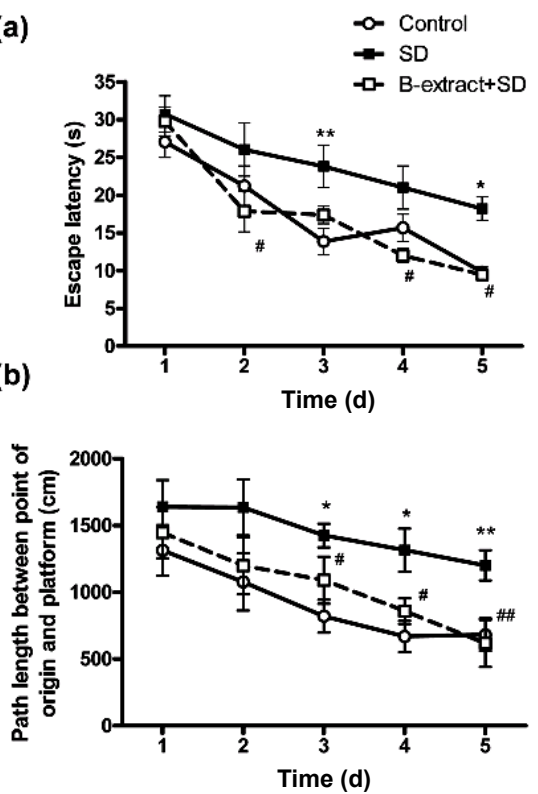

(c)

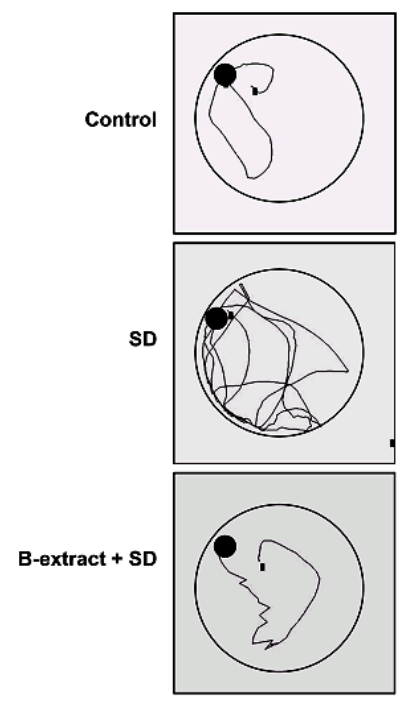

Fig. 2 Escape latency and paths during place navigation after B-extract treatment

Average escape latency (a) and path length (b) of the dementia rats were measured with a Morris water maze. Data are expressed as mean $\pm \operatorname{SEM}(n=15) .{ }^{*} P<0.05,{ }^{* *} P<0.01$ vs. control group; ${ }^{\#} P<0.05,{ }^{\# \#} P<0.01$ vs. SD group. (c) Representative swim paths of the animals. The black circle represents the platform 
of the rats in SD group were longer than those of the rats in control group $(P<0.05)$, suggesting that the rats in SD group had worse spatial memories. Compared with the rats in SD group, from Day 2, both average escape latency and path length of the rats in B-extract + SD group were significantly shortened $(P<0.05)$, which indicated that B-extract could improve spatial memory ability of dementia rats.

\subsection{Effect of B-extract on the content of $\mathrm{ACh}$, and} the activities of $\mathrm{AChE}$ and $\mathrm{ChAT}$ in brain tissue

In order to clarify the possible mechanisms of B-extract improving the spatial memory ability of dementia rats, we determined the content of neurotransmitters, which might affect memory, and the activities of key enzymes to regulate the synthesis in the hippocampus and cerebral cortex in the brain. Fig. 3 shows the determination of ACh content, ChAT activity, and AChE activity in the hippocampus and cerebral cortex of the rats. We found that the content of $\mathrm{ACh}$ in the hippocampus and cerebral cortex of rats in $\mathrm{SD}$ group is lower than that in the control group $(P<0.05)$. B-extract treatment, as expected, increases the ACh content in the hippocampus $(P<0.05)$. However, the difference in ChAT activity among the groups has no statistical significance $(P>0.05)$. Compared with the control group, AChE activity in the hippocampus and cerebral cortex of the rats in the SD group increases significantly $(P<0.001)$ and AChE activity in the hippocampus and cerebral cortex of the rats in B-extract $+\mathrm{SD}$ group is significantly lower than that in SD group $(P<0.05$ and $P<0.001$, respectively).
3.3 Effect of B-extract on the contents of $E$, NE, and DA, and the activity of MAO in brain tissue

Next we determined monoamine neurotransmitters and the activity of their key enzymes. The contents of E, NE, and DA in the hippocampus of the rats in SD group are significantly lower than those in the control group $(P<0.05)$, and the activity of MAO in the hippocampus of the rats in the SD group is significantly higher than that in the control group $(P<0.05)$. However, no significant change was observed in cortical tissue except for the DA content (Fig. 4). MAO catalyzes the deamination reaction, which is the main pathway to remove the monoamine neurotransmitters by enzymatic oxidation. Hence the decrease in monoamine neurotransmitters in the hippocampus of the rats in the SD group may be associated with increased MAO activity. At the same time, the contents of E and DA in the hippocampus of the rats in the B-extract $+\mathrm{SD}$ group are significantly higher than those in SD group $(P<0.001$ and $P<0.05$, respectively), and MAO activity is lower than that in the SD group $(P<0.05)$. It can be concluded that B-extract could increase the content of monoamine neurotransmitters in the hippocampus by reducing the activity of MAO.

\subsection{Effect of B-extract on the contents of 5-HT, Glu, and GABA in brain tissue}

In addition, we studied the content of several important excitatory and inhibitory neurotransmitters in the hippocampus and cortex. Compared with the control group, the contents of 5-HT, Glu, and GABA in the brain tissues of the rats in the SD group do not (a)

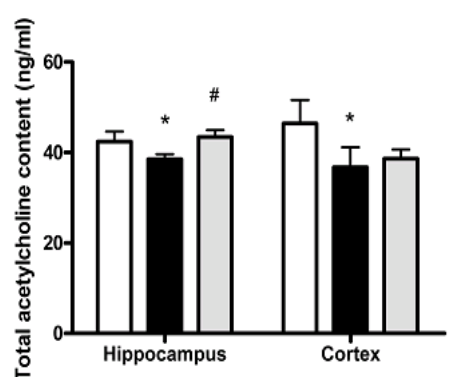

(b)

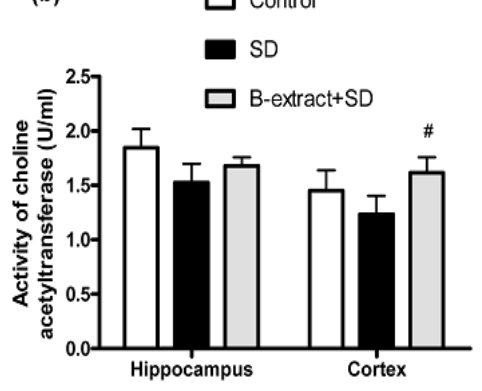

(c)

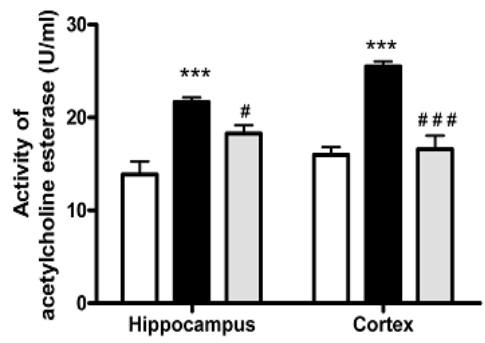

Fig. 3 Effect of B-extract on the content of ACh, the activities of ChAT and AChE in the hippocampus and cortex of dementia rats

Level of the total acetylcholine (ACh) content (a), activity of choline acetytransferase (ChAT) (b), and activity of acetylcholine esterase (AChE) (c) in the hippocampus and cortex of rats $(n=8)$. Data are expressed as mean \pm SEM. ${ }^{*} P<0.05,{ }^{* * *} P<0.001$ vs. control group; ${ }^{\#} P<0.05,{ }^{\# \#} P<0.001$ vs. SD group 
(a)

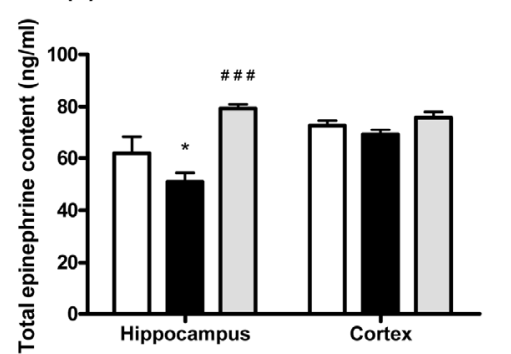

(c)

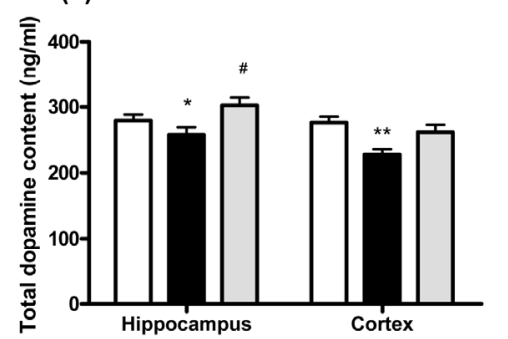

(b)

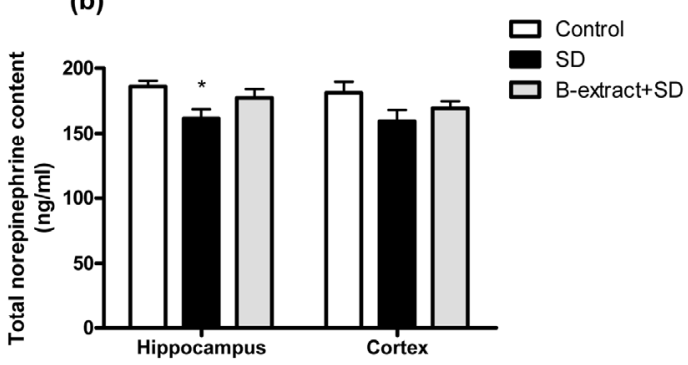

(d)

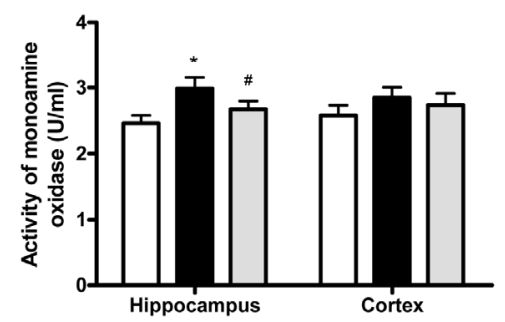

Fig. 4 Effect of B-extract on the contents of E, NE, and DA, and the activity of MAO in the hippocampus and cortex of dementia rats

The contents of the total epinephrine (E) (a), norepinephrine (NE) (b), and dopamine (DA) (c), and activity of monoamine oxidase (MAO) (d) in the hippocampus and cortex of rats $(n=8)$. Data are expressed as mean \pm SEM. ${ }^{*} P<0.05,{ }^{* *} P<0.01$ vs. control group; ${ }^{\#} P<0.05,{ }^{\# \#} P<0.001$ vs. SD group

(a)

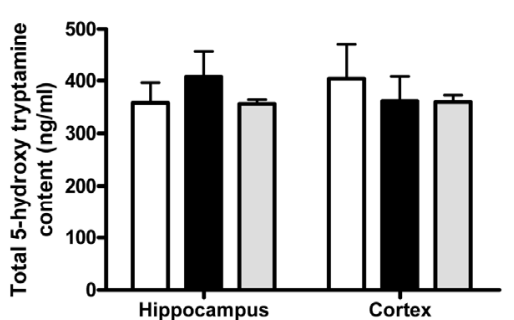

(b)

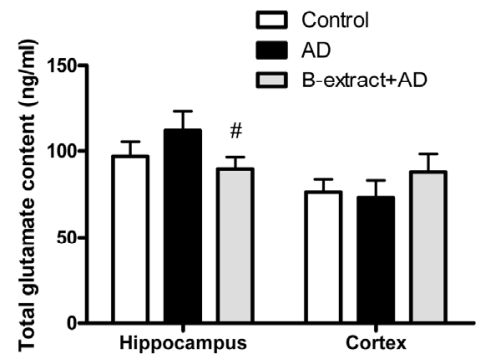

(c)

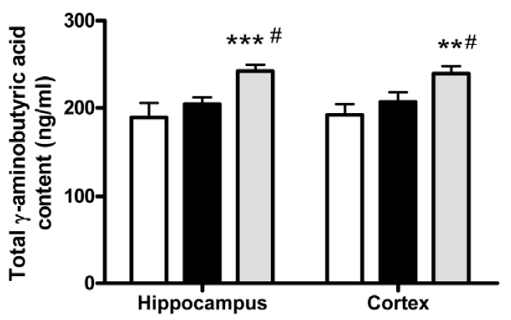

Fig. 5 Effect of B-extract on the contents of 5-HT, Glu, and GABA in the hippocampus and cortex of dementia rats The contents of the total 5-hydroxy tryptamine (5-HT) (a), glutamate (Glu) (b), and $\gamma$-aminobutyric acid (GABA) (c) in the hippocampus and cortex of rats $(n=8)$. Data are expressed as mean \pm SEM. ${ }^{* *} P<0.01,{ }^{* * *} P<0.001$ vs. control group; ${ }^{\#} P<0.05$ vs. SD group

change significantly $(P>0.05)$. After treatment with B-extract, however, the content of Glu in the hippocampus of the rats in the $\mathrm{B}$-extract+SD group is reduced by $28.6 \%(P<0.05)$ when compared with that of the SD group, while the content of GABA is increased by $18.2 \%$ in the $\mathrm{B}$-extract $+\mathrm{SD}$ group $(P<0.05$; Fig. 5$)$. Similarly, B-extract treatment also elevated the content of GABA in the cortex by $15.4 \%$.

\section{Discussion}

With the advancement of understanding of the pathogenesis of $\mathrm{AD}$, a series of animal models of dementia have been developed (Ribeiro et al., 2013; Neha et al., 2014). An ideal animal model should exhibit all AD features in animals (Neha et al., 2014). Aging animals are commonly employed in AD drug development because they mimic not only the morphological characteristics (Erickson and Barnes, 2003; Gupta et al., 2013) but also the pathophysiology (de Souza Silva et al., 2013) of human Alzheimer's disease. Chronic administration of D-gal induces changes that resemble natural aging in rodents, including neurodegeneration (Ho et al., 2003; Zhang et al., 2005) and cognitive dysfunction (Cui et al., 2006; Wang et al., 2013), and thus becomes a routinely used method to induce aging in rodents (Wei 
et al., 2005). In addition, $\mathrm{A} \beta$ plaques are a histopathological hallmark of $\mathrm{AD}$ and intrahippocampal infusion or intracerebroventricular injection of $A \beta$ has been widely applied in animal models of dementia, especially Alzheimer's disease (McLarnon and Ryu, 2008; Lopes et al., 2010). In the present study, in order to mimic the natural development of $\mathrm{AD}$, we adopted a combined administration (Qin et al., 2012) of D-gal and $A \beta_{1-42}$ to generate rat models with SD.

The typical clinical symptoms of SD include cognitive decline as well as loss and gradual deterioration of recent memory. The Morris water navigation task is widely used to study spatial learning and memory. Here, we measured spatial learning and memory of the rats by analyzing average escape latency. According to the behavioral evaluation results, the average escape latency in the SD group was longer than that in the control group, which indicated the decline of spatial memory in the dementia model.

Memory formation is associated with cholinergic neuronal function of the central nervous system, especially in the hippocampus and basal ganglia. ACh is necessary for memory formation and is the physiological basis for long-term memory. Increase in synthesis and release of $\mathrm{ACh}$ or decrease in its degradation may improve learning and memory ability (Singh et al., 2013). The synthesis of ACh is directly associated with the activity of ChAT. Studies have reported that dementia is related to the decrease in ChAT activity in the brain (Yamaguchi et al., 2002). In the present study, the content of ACh in both the hippocampus and cerebral cortex of the SD group was lower than that of the control group, while ChAT activity does not change significantly. It suggests that the main cause for the reduced content of ACh in the cholinergic neuron in dementia rats is not the decreased activity of ChAT. AChE is a hydrolase in the nervous system to degrade $\mathrm{ACh}$ and terminate related nerve impulse. Cholinergic deficit is an early finding in $\mathrm{SD}$ and $\mathrm{AChE}$ has been the most viable therapeutic target for dementia (Naaz et al., 2013). In the clinic, controlled use of AChE inhibitors has proved valuable for the treatment of dementia (Singh et al., 2013). Compared with the control group, AChE activity in the hippocampus and cerebral cortex of the rats in the SD group significantly increased while it can returned to a normal level after B-extract treatment. Taking into account that $\mathrm{AChE}$ degrades $\mathrm{ACh}$, the decreased content of ACh in SD group is likely caused by accelerated decomposition. In addition, studies have shown that there is increasing $\mathrm{AChE}$ and $\mathrm{A} \beta$ in senile plaques of AD patients (Alvarez et al., 1997). AChE and $A \beta$ may combine to form a stable compound, which is more toxic to neurons, and finally result in neuronal apoptosis (Golde et al., 2011). Therefore increased AChE activity in the brain of AD rats may produce a more toxic effect on neurons. In the present study, we found that B-extract treatment could reduce AChE activity to maintain ACh content in hippocampal neurons of the dementia rats. It suggests that B-extract improves the ability of learning and memory in dementia model rats by regulating cholinergic metabolism.

Monoamine neurotransmitters are the most important neurotransmitters in the brain, and play an irreplaceable role in the physiological activities of central nervous system. MAO catalyzes oxidative deamination of monoamine neurotransmitters such as $\mathrm{E}, \mathrm{NE}$, and DA, and generates hydrogen peroxide (Mandel et al., 2007; Hureau et al., 2010). The latter will combine with metal ions in the brain of $\mathrm{AD}$ rats to generate reactive oxygen species (ROS) (Alper et al., 1999) and cause $\mathrm{A} \beta$ deposition. Aggregation and deposition of $A \beta$ will in turn result in the increase in ROS in cells, enhance oxidative stress, and finally kill neurons (Marchi et al., 2012). Here we found that in the hippocampus, which is most closely related to learning and memory, the activity of MAO in SD group increased significantly, while the content of E, $\mathrm{NE}$, and DA decreased. We hypothesize that the increased deamination of MAO produces large amounts of hydrogen peroxide to greatly activate oxidative stress downstream and result in injury of hippocampal neurons. Interestingly, B-extract also effectively reduces the activity of MAO and increases the content of monoamine neurotransmitters in the hippocampus. Therefore, a protective effect of B-extract on neurons may be achieved through reducing the deamination of MAO and inhibiting the oxidative stress reaction.

Excitatory neurotransmitter Glu in the hippocampus may regulate the process of learning and memory through its different types of receptors and signal transduction pathways. They are essential neurotransmitters to maintain the function of the hippocampus (Peng et al., 2011). High concentration of Glu, however, produces oxidative stress toxicity 
and causes excitatory injury of neurons. The content of Glu in the hippocampus and cortex was determined in this study. We reported that although the content of Glu in SD group did not increase remarkably, B-extract could significantly reduce its content. In addition, we also measured the content of 5-HT in the hippocampus and cortex and found little effect of B-extract on the content of 5-HT. Interestingly, when the inhibitory amino acid GABA was determined, there was a great increase in the content of GABA in the hippocampal and cortex tissues, but its mechanism needs further study.

It should be mentioned that parts of our experimental findings varied in the hippocampus and cortex. It is possible that hippocampal neurons might be more vulnerable to toxicity induced by various insults than cortical neurons. Alternatively, the changes in the cortex were too small to be reliably detected by our methods.

\section{Conclusions}

B-extract has been extensively studied for its anti-oxidant activity and anti-tumor effect. However, to the best of our knowledge, the present study is the first investigation on the preventive and therapeutic effect of B-extract in the context of a SD model. In summary, the present study demonstrated that B-extract improved the learning and memory ability of dementia model rats by regulating the activities of the key enzymes for the biosynthesis and degradation of neurotransmitters, changing the contents of cholinergic, monoamine, and amino acid neurotransmitters in the hippocampus. Thus, the B-extract showed significant therapeutic effects. However, the detailed mechanism of this function needs further investigation.

\section{Compliance with ethics guidelines}

Jian-xiang LIU, Min-ying ZHU, Ci-yuan FENG, Hai-bin DING, Ying ZHAN, Zhan ZHAO, and Yue-min DING declare that they have no conflict of interest.

All institutional and national guidelines for the care and use of laboratory animals were followed.

\section{References}

Alper, G., Girgin, F.K., Ozgonul, M., et al., 1999. MAO inhibitors and oxidant stress in aging brain tissue. Eur. Neuropsychopharmacol., 9(3):247-252. [doi:10.1016/
S0924-977X(98)00035-2]

Alvarez, A., Opazo, C., Alarcon, R., et al., 1997. Acetylcholinesterase promotes the aggregation of amyloid- $\beta$ peptide fragments by forming a complex with the growing fibrils. J. Mol. Biol., 272(3):348-361. [doi:10. 1006/jmbi.1997.1245]

Checler, F., Turner, A.J., 2012. Journal of neurochemistry special issue on Alzheimer's disease: 'amyloid cascade hypothesis—20 years on'. J. Neurochem., 120(Suppl. 1): iii-iv. [doi:10.1111/j.1471-4159.2011.07603.x]

Choi, S., Park, M.S., Lee, Y.R., et al., 2013. A standardized bamboo leaf extract inhibits monocyte adhesion to endothelial cells by modulating vascular cell adhesion protein-1. Nutr. Res. Pract., 7(1):9-14. [doi:10.4162/ nrp.2013.7.1.9]

Chun, W., Johnson, G.V., 2007. The role of tau phosphorylation and cleavage in neuronal cell death. Front. Biosci., 12(1):733-756. [doi:10.2741/2097]

Cui, X., Zuo, P., Zhang, Q., et al., 2006. Chronic systemic D-galactose exposure induces memory loss, neurodegeneration, and oxidative damage in mice: protective effects of R- $\alpha$-lipoic acid. J. Neurosci. Res., 84(3):647-654. [doi:10.1002/jnr.20899]

Deguil, J., Ravasi, L., Auffret, A., et al., 2013. Evaluation of symptomatic drug effects in Alzheimer's disease: strategies for prediction of efficacy in humans. Drug Discov. Today Technol., 10(3):e329-e342. [doi:10.1016/ j.ddtec.2013.03.003]

de Souza Silva, M.A., Lenz, B., Rotter, A., et al., 2013. Neurokinin3 receptor as a target to predict and improve learning and memory in the aged organism. PNAS, 110(37):15097-15102. [doi:10.1073/pnas.1306884110]

Erickson, C.A., Barnes, C.A., 2003. The neurobiology of memory changes in normal aging. Exp. Gerontol., 38(1-2):61-69. [doi:10.1016/S0531-5565(02)00160-2]

Ferri, C.P., Prince, M., Brayne, C., et al., 2005. Global prevalence of dementia: a delphi consensus study. Lancet, 366(9503):2112-2117. [doi:10.1016/S0140-6736 (05)67889-0]

Golde, T.E., Schneider, L.S., Koo, E.H., 2011. Anti-A $\beta$ therapeutics in Alzheimer's disease: the need for a paradigm shift. Neuron, 69(2):203-213. [doi:10.1016/j. neuron.2011.01.002]

Gupta, V.K., Scheunemann, L., Eisenberg, T., et al., 2013. Restoring polyamines protects from age-induced memory impairment in an autophagy-dependent manner. Nat. Neurosci., 16(10):1453-1460. [doi:10.1038/nn.3512]

Hardy, J., 2006. Alzheimer's disease: the amyloid cascade hypothesis: an update and reappraisal. J. Alzheimers Dis., 9(Suppl. 3):151-153.

Ho, S.C., Liu, J.H., Wu, R.Y., 2003. Establishment of the mimetic aging effect in mice caused by D-galactose. Biogerontology, 4(1):15-18. [doi:10.1023/A:102241710 2206] 
Hoyert, D.L., Xu, J., 2012. Deaths: preliminary data for 2011. Natl. Vital. Stat. Rep., 61(6):1-51.

Hureau, C., Sasaki, I., Gras, E., et al., 2010. Two functions, one molecule: a metal-binding and a targeting moiety to combat Alzheimer's disease. ChemBioChem, 11(7): 950-953. [doi:10.1002/cbic.201000102]

Iqbal, K., Alonso Adel, C., Chen, S., et al., 2005. Tau pathology in Alzheimer disease and other tauopathies. Biochim. Biophys. Acta, 1739(2-3):198-210. [doi:10. 1016/j.bbadis.2004.09.008]

Lamberty, Y., Gower, A.J., Gobert, J., et al., 1992. Behavioural, biochemical and histological effects of AF64A following injection into the third ventricle of the mouse. Behav. Brain Res., 51(2):165-177. [doi:10.1016/S0166-4328(05) 80210-4]

Lee, M.J., Park, W.H., Song, Y.S., et al., 2008. Effect of bamboo culm extract on oxidative stress and genetic expression: bamboo culm extract ameliorates cell adhesion molecule expression and NFkB activity through the suppression of the oxidative stress. Clin. Nutr., 27(5):755-763. [doi:10.1016/j.clnu.2008.06.002]

Lewczuk, P., Esselmann, H., Bibl, M., et al., 2004. Tau protein phosphorylated at threonine 181 in CSF as a neurochemical biomarker in Alzheimer's disease: original data and review of the literature. J. Mol. Neurosci., 23(1-2): 115-122. [doi:10.1385/JMN:23:1-2:115]

Liu, L.T., Xu, Y., Tang, P., 2010. Mechanistic insights into xenon inhibition of NMDA receptors from MD simulations. J. Phys. Chem. B, 114(27):9010-9016. [doi:10.1021/ jp101687j]

Lopes, J.P., Oliveira, C.R., Agostinho, P., 2010. Neurodegeneration in an $\mathrm{A} \beta$-induced model of Alzheimer's disease: the role of Cdk5. Aging Cell, 9(1):64-77. [doi:10.1111/j. 1474-9726.2009.00536.x]

Mandel, S., Amit, T., Bar-Am, O., et al., 2007. Iron dysregulation in Alzheimer's disease: multimodal brain permeable iron chelating drugs, possessing neuroprotectiveneurorescue and amyloid precursor protein-processing regulatory activities as therapeutic agents. Prog. Neurobiol. 82(6):348-360. [doi:10.1016/j.pneurobio.2007.06.001]

Marchi, S., Giorgi, C., Suski, J.M., et al., 2012. MitochondriaROS crosstalk in the control of cell death and aging. $J$. Signal. Transduct., 2012:329635. [doi:10.1155/2012/329 635]

McLarnon, J.G., Ryu, J.K., 2008. Relevance of $\mathrm{A} \beta_{1-42}$ intrahippocampal injection as an animal model of inflamed Alzheimer's disease brain. Curr. Alzheimer Res. 5(5):475-480. [doi:10.2174/156720508785908874]

Naaz, H., Singh, S., Pandey, V.P., et al., 2013. Anticholinergic alkaloids as potential therapeutic agents for Alzheimer's disease: an in silico approach. Indian $J$. Biochem. Biophys., 50(2):120-125.

Neha, Sodhi, R.K., Jaggi, A.S., et al., 2014. Animal models of dementia and cognitive dysfunction. Life Sci., 109(2):
73-86. [doi:10.1016/j.lfs.2014.05.017]

Peng, S., Zhang, Y., Zhang, J., et al., 2011. Glutamate receptors and signal transduction in learning and memory. Mol. Biol. Rep., 38(1):453-460. [doi:10.1007/s11033-0100128-9]

Qin, R.A., Yao, X.X., Huang, Z.Y., 2012. Effects of Compound Danshen Tablets on spatial cognition and expression of brain $\beta$-amyloid precursor protein in a rat model of Alzheimer's disease. J. Tradit. Chin. Med., 32(1):63-66. [doi:10.1016/S0254-6272(12)60033-8]

Ribeiro, F.M., Camargos, E.R., de Souza, L.C., et al., 2013. Animal models of neurodegenerative diseases. Rev. Bras. Psiquiatr., 35(2):S82-S91. [doi:10.1590/1516-4446-20131157]

Sharma, B., Singh, N., 2010. Pitavastatin and 4'-hydroxy3'-methoxyacetophenone (HMAP) reduce cognitive dysfunction in vascular dementia during experimental diabetes. Curr. Neurovasc. Res., 7(3):180-191. [doi:10. 2174/156720210792231831]

Singh, M., Kaur, M., Kukreja, H., et al., 2013. Acetylcholinesterase inhibitors as Alzheimer therapy: from nerve toxins to neuroprotection. Eur. J Med. Chem., 70:165-188. [doi:10. 1016/j.ejmech.2013.09.050]

Song, Y., Wang, J., 2010. Overview of Chinese research on senile dementia in mainland China. Ageing Res. Rev., 9(1):S6-S12. [doi:10.1016/j.arr.2010.08.007]

Tamagno, E., Bardini, P., Guglielmotto, M., et al., 2006. The various aggregation states of $\beta$-amyloid 1-42 mediate different effects on oxidative stress, neurodegeneration, and BACE-1 expression. Free Radic. Biol. Med., 41(2): 202-212. [doi:10.1016/j.freeradbiomed.2006.01.021]

Wang, N., Chen, X., Geng, D., et al., 2013. Ginkgo biloba leaf extract improves the cognitive abilities of rats with D-galactose induced dementia. J. Biomed. Res., 27(1): 29-36. [doi:10.7555/JBR.27.20120047]

Wang, X.P., Ding, H.L., 2008. Alzheimer's disease: epidemiology, genetics, and beyond. Neurosci. Bull., 24(2):105-109. [doi:10.1007/s12264-008-0105-7]

Wei, H., Li, L., Song, Q., et al., 2005. Behavioural study of the D-galactose induced aging model in $\mathrm{C} 57 \mathrm{BL} / 6 \mathrm{~J}$ mice. Behav. Brain Res., 157(2):245-251. [doi:10.1016/j.bbr. 2004.07.003]

Wermuth, C.G., 2004. Multitargeted drugs: the end of the "One-target-one-disease" philosophy. Drug Discov. Today, 9(19):826-827. [doi:10.1016/S1359-6446(04)03213-1]

Wimo, A., Jonsson, L., Bond, J., et al., 2013. The worldwide economic impact of dementia 2010. Alzheimers Dement., 9(1):1-11.e3. [doi:10.1016/j.jalz.2012.11.006]

Xie, Q., Tang, Y., Li, W., et al., 2006. Investigation of the binding mode of (-)-meptazinol and bis-meptazinol derivatives on acetylcholinesterase using a molecular docking method. J. Mol. Model., 12(4):390-397. [doi:10. 1007/s00894-005-0058-y]

Yamaguchi, Y., Matsuno, T., Kawashima, S., 2002. Antiamnesic 
effects of azaindolizinone derivative ZSET845 on impaired learning and decreased ChAT activity induced by amyloid- $\beta 25-35$ in the rat. Brain Res., 945(2): 259-265. [doi:10.1016/S0006-8993(02)02816-0]

Zhang, Q., Li, X., Cui, X., et al., 2005. D-Galactose injured neurogenesis in the hippocampus of adult mice. Neurol. Res., 27(5):552-556. [doi:10.1179/016164105X25126]

\section{中文概要}

题 目: 竹叶提取物改善老年痴呆模型大鼠的空间学习能力

目 的: 竹叶提取物在抗氧化和抗肿瘤方面的生物学效应 已经为人所知, 但是它是否能防治痴呆还未见研 究报道。本课题旨在研究竹叶提取物对老年痴呆 大鼠的空间学习能力的影响及其可能机制。

创新点: 本研究首次提出竹叶提取物能显著改善老年痴呆 大鼠的空间学习能力, 它能通过抑制胆碱酯酶和
单胺氧化酶的活性来增加海马组织乙酰胆碱和 单胺类递质的含量, 并且调控谷氨酸和 $\gamma$-氨基丁 酸的含量, 从而正面影响老年痴呆大鼠的神经功 能。

方 法: 采用每日腹腔注射 D-半乳糖 $(60 \mathrm{mg} / \mathrm{kg}), 6$ 周末 于侧脑室注射 $\beta$-淀粉样物 (A $\beta) 1-42(10 \mu \mathrm{g})$ 的 方法制造老年痴呆大鼠模型。治疗组大鼠在造模 过程中连续 7 周每日喂饲竹叶提取物 $(20 \mathrm{mg} / \mathrm{kg})$ 。 7 周末用 Morris 水迷宫进行为期 5 天的定位航行 试验, 结束后取海马和皮质脑组织, 用酶联免疫 吸附测定法（ELISA）测定乙酰胆碱、单胺类递 质、5-羟色胺、谷氨酸和 $\gamma$-氨基丁酸含量及相关 酶活性。

结 论: 竹叶提取物可能在改善老年痴呆大鼠空间记忆能 力方面有潜在治疗价值, 其作用可能通过调控脑 内神经递质功能得以实现。

关键词: 竹叶提取物; 痴呆; 阿尔茨海默病; 神经递质 